\title{
Primordial nucleosynthesis with a varying fine structure constant: An improved estimate
}

\author{
Kenneth M. Nollett \\ Mail Code 130-33, California Institute of Technology, Pasadena, CA 91125 \\ Robert E. Lopez \\ DAMTP, University of Cambridge, Cambridge CB3 0WA, England
}

(Dated: September 9, 2021)

\begin{abstract}
We compute primordial light-element abundances for cases with fine structure constant $\alpha$ different from the present value, including many sources of $\alpha$ dependence neglected in previous calculations. Specifically, we consider contributions arising from Coulomb barrier penetration, photon coupling to nuclear currents, and the electromagnetic components of nuclear masses. We find the primordial abundances to depend more weakly on $\alpha$ than previously estimated, by up to a factor of 2 in the case of ${ }^{7} \mathrm{Li}$. We discuss the constraints on variations in $\alpha$ from the individual abundance measurements and the uncertainties affecting these constraints. While the present best measurements of primordial $\mathrm{D} / \mathrm{H},{ }^{4} \mathrm{He} / \mathrm{H}$, and ${ }^{7} \mathrm{Li} / \mathrm{H}$ may be reconciled pairwise by adjusting $\alpha$ and the universal baryon density, no value of $\alpha$ allows all three to be accommodated simultaneously without consideration of systematic error. The combination of measured abundances with observations of acoustic peaks in the cosmic microwave background favors no change in $\alpha$ within the uncertainties.

PACS numbers: 26.35.+c,24.10.-i,12.20.-m,98.80Ft,98.80.Es
\end{abstract}

\section{INTRODUCTION}

The recent literature contains claims for time variation in the fine structure constant $\alpha$ at a level of one part in $10^{5}$ since a redshift of 2 , based on observations of metal ions backlit by distant quasars [1, 2, 3, 1. Regardless of theoretical prejuduce for or against these claims [5, 6], it is of interest to examine other constraints on $\alpha$ variation. In particular, changing the fine structure constant would influence the abundances of light nuclides produced during big-bang nucleosynthesis (BBN). BBN took place at redshifts $10^{8} \leq z \leq 10^{10}$, ending minutes after the big bang, and it is the earliest cosmological test for which the basic physical laws are well-understood. The physics is less certain in varying- $\alpha$ scenarios, but BBN remains the earliest reasonable probe of variations in $\alpha$. It thus provides a large lever arm in the sense that if $\alpha$ varies with time, larger variations may plausibly (though not necessarily [7, 8) be observed over longer times.

Past BBN calculations for varying- $\alpha$ models focused on the abundance of ${ }^{4} \mathrm{He}[9$, 10, 11]. In some ways, this keeps the physics simple, because $\alpha$ should affect the ${ }^{4} \mathrm{He}$ mass fraction $Y_{P}$ only through the neutron/proton ratio at freezeout of weak interactions before BBN - any effect on the rates of subsequent nuclear reactions is irrelevant because essentially all neutrons are gathered into ${ }^{4} \mathrm{He}$ nuclei by the end of BBN. The likely size of the effect on $Y_{P}$ is also large compared with observational errors if $|\delta|>0.05$, where $\delta \equiv\left(\alpha-\alpha_{0}\right) / \alpha_{0}$ and subscript zero denotes the present value of a quantity.

More recently, Bergström, Iguri and Rubinstein [12] (hereafter BIR) extended the varying-alpha calculation to include other light nuclides by estimating the dependence of the charged-particle reaction rates on Coulomb barrier penetration and hence on $\alpha$. However, they chose to neglect several places where $\alpha$ enters the rates (from both barrier penetration and other considerations), under the assumption that the exponential $\alpha$ dependence in the Gamow factor will dominate all other $\alpha$ dependence. Our work corrects these omissions, most importantly those arising from barrier penetration and from coupling of photons to nuclear currents. We also estimate the effects of final-state barrier penetration and the $\alpha$ dependence of the nuclear masses. We use more elaborate models for the $\alpha$ dependences of the mass-7 radiative captures, and we correct BIR's use of reaction rates that were already out of date when they did their work.

Finally, we elaborate the BIR discussion of BBN constraints on $\alpha$, examining the region of the $\left(\Omega_{B} h^{2}, \alpha\right)$ plane allowed by each of the observed light-nuclide abundances. $\left(\Omega_{B}\right.$ is the universal baryon density in units of the critical density; $h$ is the Hubble constant in units of $100 \mathrm{~km} \mathrm{~s}^{-1} / \mathrm{Mpc}$.) We then discuss the prospects for producing a consistent solution, given systematic uncertainties in the observed abundances. We leave aside the question of how (or whether) other parameters of the standard model of particle physics vary as $\alpha$ varies [9, 11, 13, 14, 15, 16], though this may also affect BBN.

\section{LIGHT NUCLEI AND THE FINE STRUCTURE CONSTANT}

The BBN light-element abundances, apart from ${ }^{4} \mathrm{He}$, depend on the fine structure constant through nuclear reaction rates. Calculating how these rates depend on $\alpha$ is the main task undertaken by BIR.

Charged particles must tunnel through a Coulomb barrier to react at low energies, and this produces most of the energy dependence in the cross section. Changing $\alpha$ 
raises or lowers the Coulomb barrier, altering the reaction rates. To describe laboratory results and compute thermally-averaged rates, it is useful to separate out the Coulomb part of the energy dependence by writing the low-energy cross section as

$$
\sigma(E)=\frac{S(E)}{E} e^{-2 \pi \eta} .
$$

Here, $\eta=\alpha Z_{1} Z_{2} \sqrt{\mu / 2 E}, \mu$ is the reduced mass, and $Z_{1}$, $Z_{2}$ are the charges of the incoming nuclei. The astrophysical $S$-factor $S(E)$ is then usually a slowly-varying function of energy.

BIR calculate the $\alpha$ dependences of the thermal reaction rates that result if the $S$-factors are assumed to be independent of $\alpha$; i.e., if all of the $\alpha$ dependence is expressed in Eqn. 1 and the definition of $\eta$. We have verified that their results are correct, given this assumption. However, Eqn. 11 does not explicitly contain all of the expected $\alpha$ dependence, even if the only effect of changing alpha is to change the Coulomb barrier. Moreover, BBN takes place at relatively high energies (a few hundred $\mathrm{keV}$ ) and $Z_{1} Z_{2}$ is only in the range $1-4$ in $\mathrm{BBN}$, so the exponential does not dominate sufficiently to make $\alpha$ dependence of the $S$-factor completely negligible by comparison. If $\mathrm{BBN}$ involved energies a factor of two lower or nuclei with only slightly larger charges, corrections to the BIR treatment would be negligible.

The first source of $\alpha$ dependence in $S(E)$ is Coulomb barrier penetration. Cross sections are proportional to the barrier penetration factor $v_{l}$ :

$$
v_{l}=\frac{1}{F_{l}^{2}(\eta, k R)+G_{l}^{2}(\eta, k R)} .
$$

Here, $F_{l}$ and $G_{l}$ are the regular and irregular Coulomb wave functions, respectively [17], $l$ is orbital angular momentum of the entrance channel, $k$ is the wavenumber in the entrance channel, and $R$ is the nuclear interaction radius. (A clear derivation of Eqn. 2 may be found in Chapter VIII of Blatt and Weisskopf [18]). Assuming $l=0$ (a reasonable assumption at very low energies), $v$ is nonzero even when $R=0$. For small $k$, it is reasonable to approximate the barrier-penetration part of the cross section as

$$
\begin{aligned}
\sigma(E) & \propto v_{l} \\
& =\left[G_{0}(\eta, 0)\right]^{-2} \\
& =\frac{2 \pi \eta}{e^{2 \pi \eta}-1} \\
& \approx 2 \pi \alpha Z_{1} Z_{2} \sqrt{\mu / 2 E} e^{-2 \pi \eta}
\end{aligned}
$$

We have thus "derived" the heuristic expression in Eqn. 1, and found that $S(E)$ is proportional to $\alpha$. If the entrance channels of all reactions are reasonably approximated as $S$ waves penetrating to zero separation, it follows that the charged-particle rates in BIR should be multiplied by an additional factor of $(1+\delta)=\alpha_{\mathrm{BBN}} / \alpha_{0}$ (where $\alpha_{\mathrm{BBN}}$ is the value of $\alpha$ at the time of $\mathrm{BBN}$ ). When
$R>0$ or $l>0$, Eqn. 1 almost always provides an overestimate of the sensitivity of Eqn. 2 to $\alpha$. (Exceptions with $\sim 10 \%$ underestimates of $d v_{l} / d \alpha$ occur at $E>100$ $\mathrm{keV}$ for reactions with $Z_{1} Z_{2}=1$.)

The pedagogical literature usually motivates Eqn. 11 by a WKB approximation. Even where WKB provides a quantitatively good approximation (not the usual case [18]), the factor of $\alpha$ on the front of Eqn. 1 enters the WKB barrier penetration factor through the normalization that matches solutions across the classical turning point.

When a reaction produces two charged particles, they must escape from a Coulomb barrier, and this introduces more $\alpha$ dependence in the rates. The cross section is again suppressed by a factor of the form shown in Eqn. 2, but with $E \rightarrow Q+E$ and appropriate charges. The effect is weak because $Q$ (the energy released) is generally much larger than the Coulomb barrier, but there are two exceptions, ${ }^{3} \mathrm{He}(n, p){ }^{3} \mathrm{H}$ and ${ }^{7} \mathrm{Be}(n, p){ }^{7} \mathrm{Li}$. These two cases are very interesting, because their $Q$ values depend strongly on $\alpha$ (see below), and this introduces $\alpha$ dependence in the final-state barrier penetration that almost cancels the dependence due to the changing barrier height.

The generally high $Q$-values in BBN invalidate the approximation in Eqn. 4 for final states. In fact, the final-state barrier penetration factors for the $\mathrm{BBN}$ reactions are almost independent of energy over the BBN energy range. Even Eqn. 3 badly overestimates the effect because $k$ is larger for the final states. We estimate the final-state suppression by assuming $R=2 \mathrm{fm}$ and $l=0,1,2$, computing logarithmic derivatives of $v_{l}$ with respect to alpha, and looking for the largest effect. The results should be rough but reasonable upper limits to the size of the effect. To keep thermal rates simple, we exploit the weak energy dependence of these derivatives and multiply the rates by energy-independent factors $(1+a \delta)$ which model their $\alpha$ dependence. In these factors, $a$ is estimated to be slightly larger in magnitude than $\alpha v_{l}^{-1} d v_{l} / d \alpha$ for all $l$ considered, and the adopted values are shown in Table 1 . In the cases of ${ }^{3} \mathrm{He}(n, p)^{3} \mathrm{H}$ and ${ }^{7} \mathrm{Be}(n, p)^{7} \mathrm{Li}$, the low $Q$-value leads us to include in $a$ the complete dependence of final-state channel widths on $Q$. (Channel widths are proportional to the product of channel velocity and $v_{l}$ [18], both of which vary with Q.) This adjustment is based on our estimate of the electromagnetic contribution to the masses (see below) and takes the corresponding numbers in Table from slightly negative to slightly positive.

The matrix elements for radiative captures - photonemitting processes - are proportional to $\alpha$ because $\alpha$ is the strength of the coupling between the photon and nuclear currents. Therefore, all radiative capture rates should be multiplied by an additional factor of $(1+\delta)$.

Some of the radiative captures $\left[{ }^{3} \mathrm{H}(\alpha, \gamma)^{7} \mathrm{Li}\right.$ and $\left.{ }^{3} \mathrm{He}(\alpha, \gamma){ }^{7} \mathrm{Be}\right]$ are also external reactions (e.g. 19, 201), so particles need not penetrate the Coulomb barrier all the way to the nuclear surface; the reaction matrix ele- 


\begin{tabular}{cc}
\hline \hline Reaction & $a$ \\
\hline$d(d, p)$ & -0.16 \\
${ }^{3} \operatorname{He}(n, p)$ & +0.30 \\
${ }^{3} \operatorname{He}(d, p)$ & -0.09 \\
${ }^{7} \operatorname{Li}(p, \alpha)$ & -0.18 \\
${ }^{7} \operatorname{Be}(n, p)$ & +0.20 \\
\hline \hline
\end{tabular}

TABLE I: Corrections for final-state interactions for processes involving two charged particles in the final state. To correct the rates, multiply by $(1+a \delta)$, where a is given above.

ments contain large contributions from outside the nuclear interaction radius. Further, Coulomb forces govern the forms of the final states $\left({ }^{7} \mathrm{Li}\right.$ and ${ }^{7} \mathrm{Be}$ bound states) at these distances. Most importantly, the electrostatic energies of the bound states are also large fractions of the reaction $Q$ values, so that variations in $\alpha$ change the outgoing photon energy significantly. This energy enters the cross sections as $\sigma \propto E_{\gamma}^{3}$. We used a simple radiative capture model to estimate the combined size of these three effects, though we found that the last one dominates.

We assume the following: 1) These reactions can be treated as direct captures in which the bound states consist of smaller clusters: ${ }^{4} \mathrm{He}$ and ${ }^{3} \mathrm{H}$ in the case of ${ }^{7} \mathrm{Li}$, or ${ }^{4} \mathrm{He}$ and ${ }^{3} \mathrm{He}$ in the case of ${ }^{7} \mathrm{Be}$. 2) Wave functions can be generated from potential models; we use the potential of Buck et al. 21]. 3) The only effect of changing $\alpha$ is to change the charges in the Coulomb term of this potential. There is no question that the assumptions numbered 1 and 2 are reasonable descriptions of laboratory data. It is plausible that assumption number 3 is no worse than other assumptions we have made implicitly in modelling BBN with varying $\alpha$. We estimate the variation of the thermal reaction rates with $\alpha$ from this model. We find the effect on the thermal rates (beyond the effect in Eqn. 1) to be roughly independent of temperature, and we characterize it by fits to the computed variation beyond the corrections already discussed,

$$
f=\left(\frac{\alpha}{\alpha_{0}}\right)^{-2} \frac{\langle\sigma(\alpha) v\rangle}{\left\langle\sigma\left(\alpha_{0}\right) v\right\rangle}
$$

(with $\langle\sigma v\rangle$ the thermally-averaged rate). These fits give estimates of the rates at $\alpha \neq \alpha_{0}$ that are accurate to $20 \%$ in the additional effect beyond the exponential factor and two powers of $\alpha$ already considered (at temperatures less than $10^{10} \mathrm{~K}$ ), and they are shown in Table II. We assume that this $\alpha$ dependence holds relative to the reaction rates computed from laboratory measurements. (See Sec. III for the baseline $\alpha=0$ rates.)

Nuclear masses depend on $\alpha$ through electromagnetic interactions between nucleons. In turn, differences between initial-state and final-state masses are used in nucleosynthesis calculations to determine the rates of reverse reactions (by detailed balance). BIR report that reverse reactions have essentially no effect on the BBN yields, but we find that when we alter the masses, the

\begin{tabular}{lcccccc}
\hline \hline Reaction & $a_{0}$ & $a_{1}$ & $a_{2}$ & $a_{3}$ & $a_{4}$ & $a_{5}$ \\
\hline${ }^{3} \mathrm{H}(\alpha, \gamma){ }^{7} \mathrm{Li}$ & 1 & 1.3718 & 0.502 & 0.1829 & 0.2693 & -0.2182 \\
${ }^{3} \mathrm{He}(\alpha, \gamma){ }^{7} \mathrm{Be}$ & 1 & 2.148 & 0.6692 & -5.566 & -10.63 & -5.730 \\
\hline \hline
\end{tabular}

TABLE II: Estimated dependence of radiative capture rates on $\alpha$, with $f(\alpha)=\sum a_{i} \delta^{i}$ as defined in Eqn. .

TABLE III: Electromagnetic contributions $\Delta M_{E M}$ to the reaction energy yields, from the calculations of Refs. 22, 23]. Shown for comparison are the total energy yields $Q_{0}$.

\begin{tabular}{ccc}
\hline \hline Reaction & $\Delta M_{E M}(\mathrm{MeV})$ & $Q_{0}(\mathrm{MeV})$ \\
\hline$p(n, \gamma) d$ & -0.018 & 2.224 \\
$d(p, \gamma){ }^{3} \mathrm{He}$ & -0.70 & 5.4940 \\
$d(d, p) t$ & -0.004 & 4.033 \\
$d(d, n){ }^{3} \mathrm{He}$ & -0.682 & 3.270 \\
$t(d, n){ }^{4} \mathrm{He}$ & -0.81 & 17.590 \\
${ }^{3} \mathrm{He}(d, p){ }^{4} \mathrm{He}$ & -0.81 & 18.353 \\
$t(\alpha, \gamma){ }^{7} \mathrm{Li}$ & -0.844 & 2.467 \\
${ }^{3} \mathrm{He}(\alpha, \gamma){ }^{7} \mathrm{Be}$ & -1.74 & 1.587 \\
${ }^{3} \mathrm{He}(n, p){ }^{3} \mathrm{H}$ & 0.678 & 0.7637 \\
${ }^{7} \mathrm{Li}(p, \alpha){ }^{4} \mathrm{He}$ & 0.016 & 17.3459 \\
${ }^{7} \mathrm{Be}(n, p){ }^{7} \mathrm{Li}$ & 1.574 & 1.6443 \\
\hline \hline
\end{tabular}

yields change at $\Omega_{B} h^{2}>0.025$.

We adopt electromagnetic contributions $M_{E M}$ to nuclear masses based on quantum Monte Carlo calculations [22, 23], where all electromagnetic contributions (including magnetic-moment interactions) are computed explicitly. We then multiply $M_{E M}$ by $(1+\delta)$ to vary $\alpha$, and adjust the Q-values accordingly to obtain $Q=$ $Q_{0}+\Delta M_{E M} \delta$. The adopted values of $\Delta M_{E M}$ are given in Table III.

In summary, to calculate BBN yields for an altered fine structure constant, first apply the changes described in BIR. Then apply these additional corrections:

- Coulomb normalization factor: Multiply all charged-particle rates by a factor of $(1+\delta)$.

- Radiative captures: Multiply rates for radiative captures by another factor of $(1+\delta)$. Multiply the rates for ${ }^{3} \mathrm{He}(\alpha, \gamma){ }^{7} \mathrm{Be}$ and ${ }^{3} \mathrm{H}(\alpha, \gamma){ }^{7} \mathrm{Li}$ by the additional factors $f$ in Table III.

- Final-state Coulomb interactions: Multiply rates with two charged particles in the final state by a factor $(1+a \delta)$, using the values of $a$ given for each

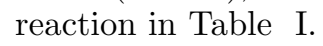

- Masses: Adjust the reaction $Q$-values using the electromagnetic contributions shown in Table III: $Q=Q_{0}+\Delta M_{E M} \delta$.

Most of these effects have the opposite sign from the exponential factor considered in BIR. We consequently expect a weaker dependence on $\alpha$ for the reaction rates and resulting BBN yields. 
The uncertainty in this improved treatment is difficult to assess. The adjustment of the $Q$-values is relatively model-independent. Adjustment of the rates, on the other hand, is less certain because we have used approximations to the Coulomb penetrabilities, and also because we have neglected everything happening inside the nucleus. The result is certainly better (and closer to selfconsistency) than the overestimate that results from assuming that $S$-factors are independent of $\alpha$. Our approximations to barrier penetrabilities almost always overestimate the effect of varying $\alpha$. The overestimates are generally $5-10 \%$ of the variation, except where $Z_{1} Z_{2}=1$; here, they may be underestimates by up to $10 \%$ of the variation. These percentages are relative to barrier penetration factors for $R=2 \mathrm{fm}$ and $l<2$, and not relative to the (unknown and perhaps unknowable) total change in the cross section when $\alpha$ changes. The most extreme limit to the validity of the method is where states become unbound, i.e, $Q=Q_{0}+\Delta M_{E M} \delta=0$. This occurs at about $\delta=0.5$ for the first excited state of ${ }^{7} \mathrm{Be}$, although our radiative capture calculation fails for numerical reasons beyond about $\delta=0.3$. Our treatment of final-state barrier penetration also assumes that $\delta$ is "small" (being based on first derivatives), but these corrections turn out to be relatively unimportant. We assume symmetric limits to the validity of our analysis, $-0.3<\delta<0.3$, with less-questionable validity at $-0.1<\delta<0.1$.

\section{LABORATORY RATES}

We note finally that BIR used reaction rates that were already out of date, and they did not cite sources for the rates they did use. All of their important rates come from the evaluation by Smith, Kawano, and Malaney (SKM) 24 (and the others trace to the Caughlan and Fowler compilations [25]). In the time since SKM (1993), some of the most uncertain cross sections have been remeasured. Newer, better rates exist for the processes $d(p, \gamma)^{3} \mathrm{He}$ [26, 27], $t(\alpha, \gamma)^{7} \mathrm{Li}$ [28, and ${ }^{3} \mathrm{He}(n, p)^{3} \mathrm{H}$ [29]. The revised rate for the first reaction has the usual functional form derived by the $S$-factor formalism (though its parameters are actually fitted to a numerical integration), and the expression from BIR, as corrected in Sec. II, may plausibly provide its $\alpha$ dependence. We have thus substituted the coefficients from Ref. [27] into the BIR expression.

For the second reaction, the revised rate is again a fit to a numerical integration, but the functional form used does not show up in the BIR calculation. Since we have a physical model for the variation of this rate with $\alpha$, and the BIR formalism does in fact account for much of the $\alpha$ dependence in this model, we adopt the following somewhat convoluted procedure: we first computed a fitting formula for the ratio of the revised rate to the SKM rate. In our BBN code, we multiply the BIR $\alpha$-dependent rate by this fitting formula. We then apply the corrections from Sec. II. The result provides both the correct, up-to-date rate at $\alpha=\alpha_{0}$, and the $\alpha$ dependence computed from our direct capture model. The SKM $S$-factor contains a term of the form

$$
S(E)=S(0) q(E) e^{-\beta E},
$$

which we drop in this procedure to more accurately reproduce the $\alpha$ dependence computed in our model. This is not a problem with the SKM rate evaluation, but rather a question of producing simple, adequate fitting functions for the $\alpha$ dependence of the rate. (Note that in principle, this term arises from the external-capture nature of the reaction, so that $\beta$ should almost certainly depend on $\alpha$ - a possibility not considered in BIR.)

Finally, we updated the rate for ${ }^{3} \mathrm{He}(n, p)^{3} \mathrm{H}$, which does not need the BIR treatment because of the neutron in the entrance channel. In summary, our calculations used the SKM rates, with $\alpha$ variation estimated as described above, and with updates from Refs. [27, 28, 29. We compared a calculation using these rates and $\alpha_{\mathrm{BBN}}=\alpha_{0}$ with the corresponding results from the NACRE [30] and Nollett \& Burles 31] evaluations of the rates (which are not suited to computing $\alpha$ variation). The results were in agreement within the uncertainties.

To compare our calculation with observed abundances, it will be necessary to take uncertainties on the laboratory measurements into account. The effects of these uncertainties on the computed abundances can, in general, only be estimated by Monte Carlo methods. Since we compute a large grid of models in $\Omega_{B} h^{2}$ and $\alpha$, such a procedure would be time-consuming. Instead, we make very simple, conservative estimates of the uncertainties. The uncertainties on the computed helium mass fraction $Y_{P}$ have been estimated by Lopez \& Turner [32] to be \pm 0.0004 from experiment and \pm 0.0002 from theory. We have not included their elaborate treatment of small effects in our calculation, and we will assume an error of 0.0005 on the calculation. For $\Omega_{B} h^{2}>0.011$, the uncertainty in $\mathrm{D} / \mathrm{H}$ arises mainly from the rate for $d(p, \gamma)^{3} \mathrm{He}$; we use an estimate of $9 \%$ here, based on the uncertainty in $\mathrm{D} / \mathrm{H}$ at $\Omega_{B} h^{2}=0.036$, its maximum in Ref. 31. Similarly, the uncertainty in ${ }^{7} \mathrm{Li} / \mathrm{H}$ at $\Omega_{B} h^{2}>0.011$ is governed by the uncertainty in the rate for ${ }^{3} \mathrm{He}(\alpha, \gamma){ }^{7} \mathrm{Be}$. We take as the uncertainty in ${ }^{7} \mathrm{Li} / \mathrm{H}$ its maximum of $0.05 \mathrm{dex}$ at $\delta=0$ from Ref. 31. We will see below that ${ }^{3} \mathrm{He} / \mathrm{H}$ depends only weakly on $\alpha_{\mathrm{BBN}}$. Moreover, the identification of the observed ${ }^{3} \mathrm{He} / \mathrm{H}$ with the primordial value 33. seems to us problematic, so we will not use ${ }^{3} \mathrm{He}$ in our examination of the evidence for varying $\alpha$.

\section{RESULTS}

We incorporated all of the $\alpha$ dependence discussed above into the standard BBN code 34, 35 and computed yields. Figure 11 shows the resulting light-element abundances for $\delta=0, \pm 0.05$, compared with a calculation applying the BIR formalism to updated laboratory rates. For $\mathrm{D} / \mathrm{H}$ and ${ }^{7} \mathrm{Li} / \mathrm{H}$, we find that the $\alpha$ dependence 


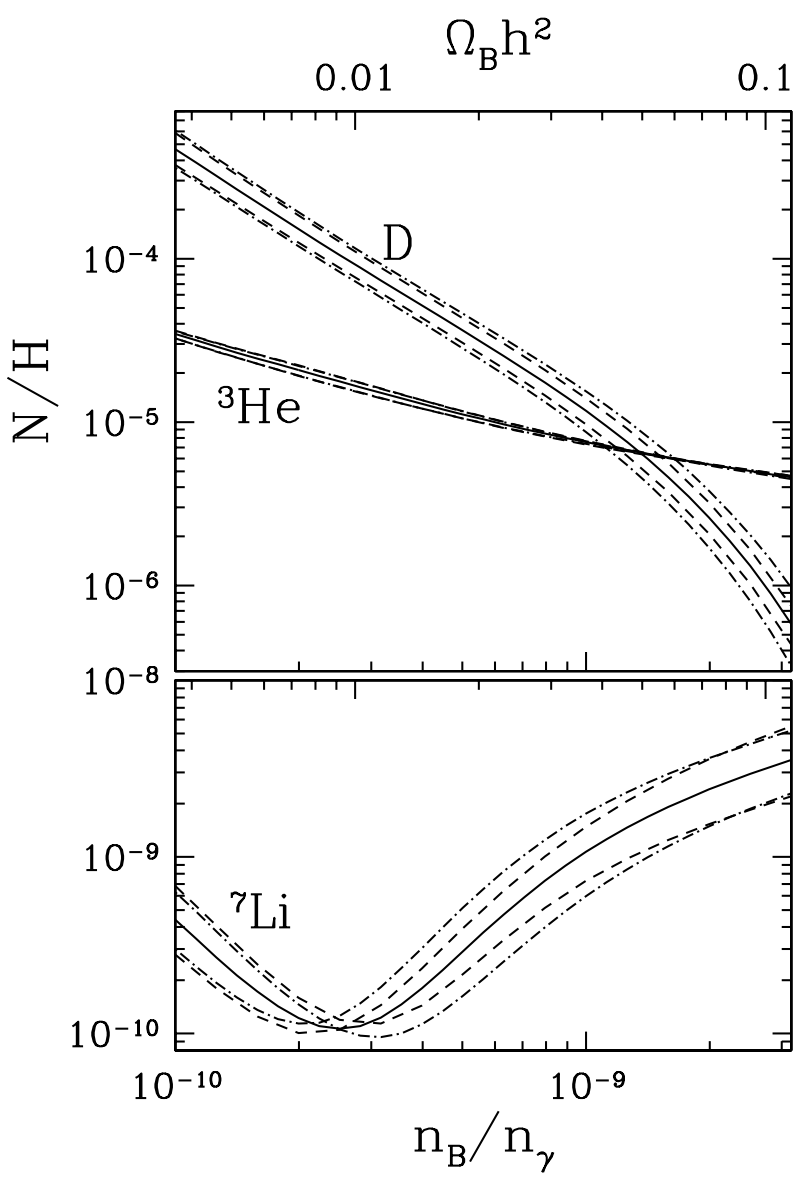

FIG. 1: Light element abundances for $\delta=0$ (solid lines), $\delta= \pm 0.05$ from the BIR method (dash-dotted lines) and $\delta=$ \pm 0.05 from our calculation (dashed lines). $n_{B}$ and $n_{\gamma}$ are the number densities of baryons and photons, respectively.

is weaker than that found by BIR, though the effect still works in the same direction. This can be understood as follows: the strongest $\alpha$ dependence in their calculation is in the suppression of charged-particle rates by the negative exponential in those rates. In our calculation, positive powers of $\alpha$ from current coupling and careful treatment of the barrier penetration partially cancel this suppression. The ${ }^{7} \mathrm{Li}$ also receives a somewhat smaller contribution from adjustments in outgoing photon energy which we modelled. Effects of the changing $Q$ values on the reverse reactions become important for ${ }^{7} \mathrm{Li} / \mathrm{H}$ at large $\Omega_{B} h^{2}$, making our estimate coincidentally equal to that of BIR at $\Omega_{B} h^{2}>0.065$. Our estimate of final-state effects makes a much smaller contribution to the yields than any of the other corrections we have added to the calculation.

Our results for the ${ }^{4} \mathrm{He}$ abundance agree with BIR. This abundance depends on $\alpha$ primarily through the neutron-proton mass difference $m_{n}-m_{p}$ which governs the neutron/proton ratio at the start of BBN. Like BIR, we use the phenomenological prescription of Gasser and Leutwyler [36] to model the $\alpha$ dependence of the nucleon

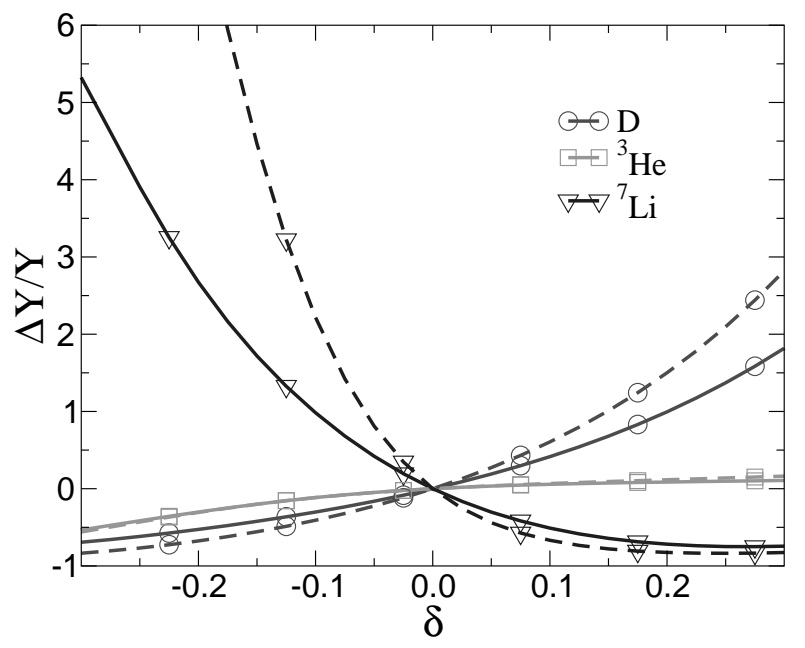

FIG. 2: Fractional change in yields from their value at $\delta=0$, computed at $n_{B} / n_{\gamma}=5.6 \times 10^{-10}\left(\Omega_{B} h^{2}=0.0204\right)$. Solid curves are from our calculation. Dashed curves indicate results from the methods of BIR.

mass splitting:

$$
m_{n}-m_{p}=1.3(1+c \delta) \mathrm{MeV},
$$

where $c=-0.59$. As pointed out in BIR, this model is plausible, but certainly not definitive, so the ${ }^{4} \mathrm{He}$ yields are less certain than those of other light nuclides. We use the Gasser \& Leutwyler value because we did not find competing, explicit values of $c$ in the literature. The abundances of the other nuclides are for the most part affected only weakly by this uncertainty, but the nucleon mass splitting makes an important contribution to the $\alpha$ dependence of ${ }^{7} \mathrm{Li} / \mathrm{H}$ at low $\Omega_{B} h^{2} \leq 0.010$. We show the dependences of $\mathrm{D} / \mathrm{H},{ }^{3} \mathrm{He} / \mathrm{H}$, and ${ }^{7} \mathrm{Li} / \mathrm{H}$ on $\delta$ at $\Omega_{B} h^{2}=$ 0.0204 in Fig. 2 .

\section{OBSERVATIONAL CONSTRAINTS}

Given observationally-inferred primordial abundances, our calculations can be used to constrain possible combinations of $\Omega_{B} h^{2}$ and $\delta$. In principle, two abundances suffice to fix values of the two parameters, and a third abundance provides a check on the result. In reality, there are systematic errors associated with both the measurements and the varying- $\alpha$ calculation, and we find no solution which satisfies the $\mathrm{D},{ }^{4} \mathrm{He}$, and ${ }^{7} \mathrm{Li}$ observations simultaneously. $\left({ }^{3} \mathrm{He} / \mathrm{H}\right.$ shows relatively weak dependence on $\alpha$; it may in principle help constrain $\Omega_{B} h^{2}$ so that $\alpha$ may be extracted from other abundances, given the primordial ratio.) We now examine the constraints provided by the present observations. This discussion is in large part supplementary to those in BIR and in Avelino et al. 37] (who used the BIR rates, but also applied statistical error estimation to a subset of the data). However, we also draw upon our improved estimation of the yields. The 


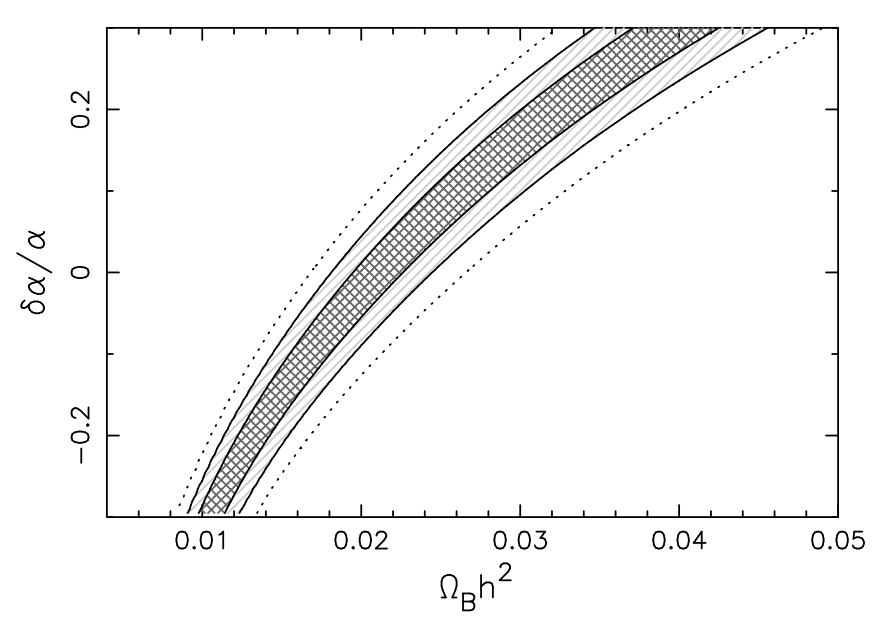

FIG. 3: Regions of $1 \sigma, 2 \sigma$, and $3 \sigma$ confidence, based on the $\mathrm{D} / \mathrm{H}$ measurement of O'Meara et al. 40].

reader should keep in mind that the primordial lightnuclide abundances require difficult observational work and are by no means settled questions; we will attempt to point out the most serious problems, but the reader is referred to recent reviews of the topic for critical discussion of the uncertainties and appropriate caveats 38, 39. It what follows, we assume Gaussian distributions for all uncertainties and include the roughly-estimated yield uncertainties of Sec. III.

A value for a single light-nuclide abundance specifies one constraint relating $\Omega_{B} h^{2}$ and $\delta$, and therefore defines a curve in the $\left(\Omega_{B} h^{2}, \delta\right)$ plane. As a first example, we show in Fig. 3 the constraint in the $\left(\Omega_{B} h^{2}, \delta\right)$ plane provided by an average of the extragalactic $\mathrm{D} / \mathrm{H}$ measurements of Tytler and collaborators, $(3.0 \pm 0.4) \times 10^{-5}$ [40. (There exist measurements by other groups, which we exclude because of concerns about signal-to-noise 441 and complex velocity structure 42, 43]; these tend to increase the scatter.) The largest weakness of $\mathrm{D} / \mathrm{H}$ as a probe of $\mathrm{BBN}$ is the difficulty of obtaining large enough numbers of high-quality data to be confident in their analysis. Because there is uncertainty in the observations, there is uncertainty in specifying the curve; this is indicated by shaded regions delimiting 1,2 , and $3 \sigma$ confidence levels. We note that the error estimate of Ref. 40 is rather conservative.

The analogous curves based on halo-star observations of ${ }^{7} \mathrm{Li}$ are shown in Fig. 14. We derive these curves from the primordial ${ }^{7} \mathrm{Li}$ abundance advocated by Ryan 44 and Ryan et al. 45, $\log _{10}\left({ }^{7} \mathrm{Li} / \mathrm{H}\right)=-9.91_{-0.13}^{+0.19}$. It should be kept in mind that there could be factor-oftwo effects associated with $\mathrm{Li}$ destruction in the observed stars 46, 47, 48 and with the stellar atmosphere models used to derive abundances 49 . There are two curves for ${ }^{7} \mathrm{Li} / \mathrm{H}$, reflecting the two paths for its production in BBN: a low $-\Omega_{B}$ case in which ${ }^{7} \mathrm{Li}$ is produced directly, and a high- $\Omega_{B}$ case in which ${ }^{7} \mathrm{Li}$ is produced as ${ }^{7} \mathrm{Be}$, followed by beta decay. Again, confidence intervals are indicated by the shaded regions. Comparison of Figs. 3

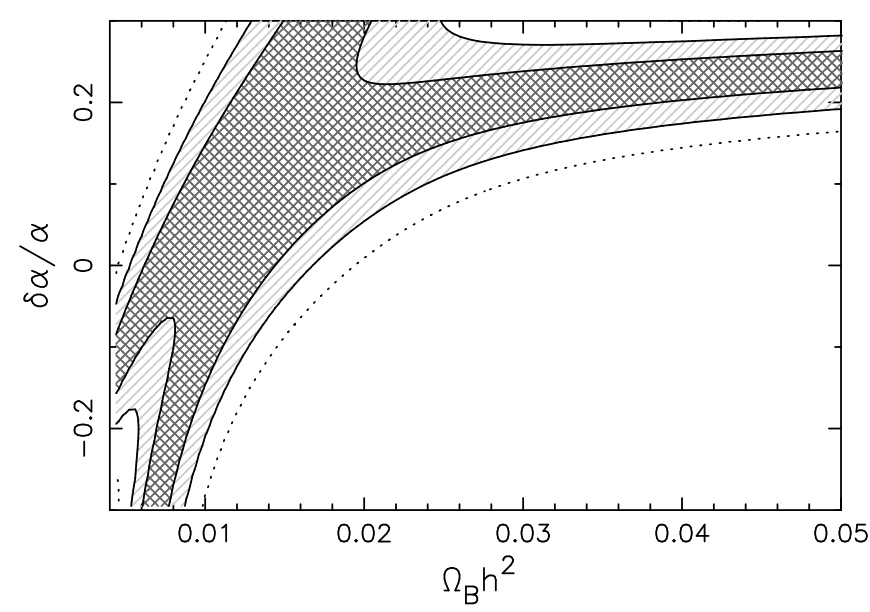

FIG. 4: Regions of $1 \sigma, 2 \sigma$, and $3 \sigma$ confidence, based on the ${ }^{7} \mathrm{Li} / \mathrm{H}$ value from Ryan 44 .

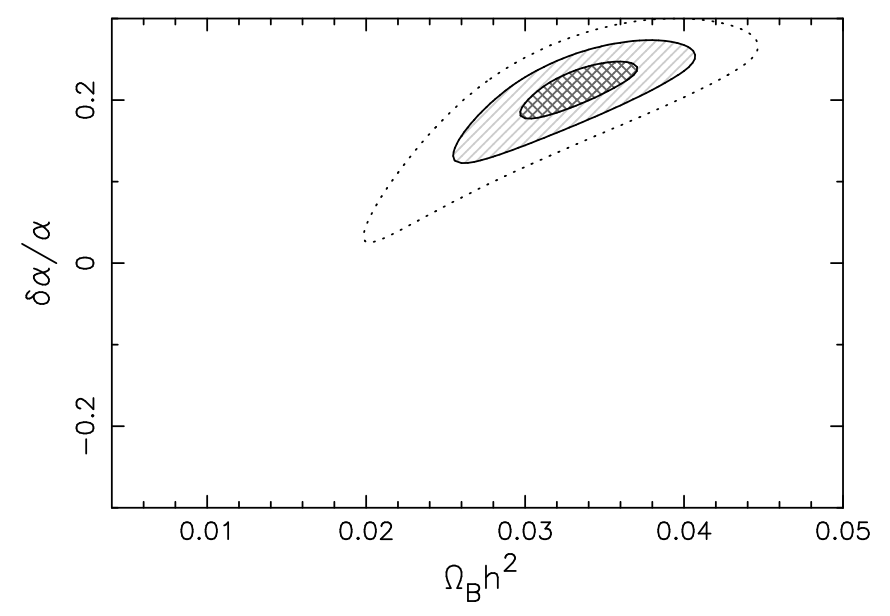

FIG. 5: Confidence levels in the $\left(\Omega_{B} h^{2}, \delta\right)$ plane for $\mathrm{D} / \mathrm{H}$ and ${ }^{7} \mathrm{Li} / \mathrm{H}$ combined, ignoring ${ }^{4} \mathrm{He}$. The contours reflect $1 \sigma, 2 \sigma$, and $3 \sigma$ confidence levels. The solution for these two parameters given the two constraints is $\Omega_{B} h^{2}=0.034, \delta=0.23$, but the result fails a check against a third constraint.

and 4 shows one point where the $\mathrm{D} / \mathrm{H}$ and ${ }^{7} \mathrm{Li}$ results can be reconciled. This point occurs at $\Omega_{B} h^{2}=0.034$ and $\delta=0.23$. Although we consider the range of validity of our treatment to be $-0.3<\delta<0.3$, there does appear to be a second solution at lower $\Omega_{B} h^{2}$ and an extreme negative $\delta$. Note that the best-fit model for $\mathrm{D}$ and ${ }^{7} \mathrm{Li}$ with $\delta=0$ is rejected at $98 \%$ confidence. The joint constraint is shown in Fig. 5 .

How seriously should this result be taken? A solution can always be found for two abundances [assuming that the curves in the $\left(\Omega_{B} h^{2}, \alpha\right)$ plane intersect], so its validity must be checked with a third abundance. The other abundance at our disposal is that of ${ }^{4} \mathrm{He}$, as observed in extragalactic HıI regions. Here, we confront unavoidable systematic problems associated with the extraction of abundances from spectral-line data. The two largest compilations of data yield discrepant values for 

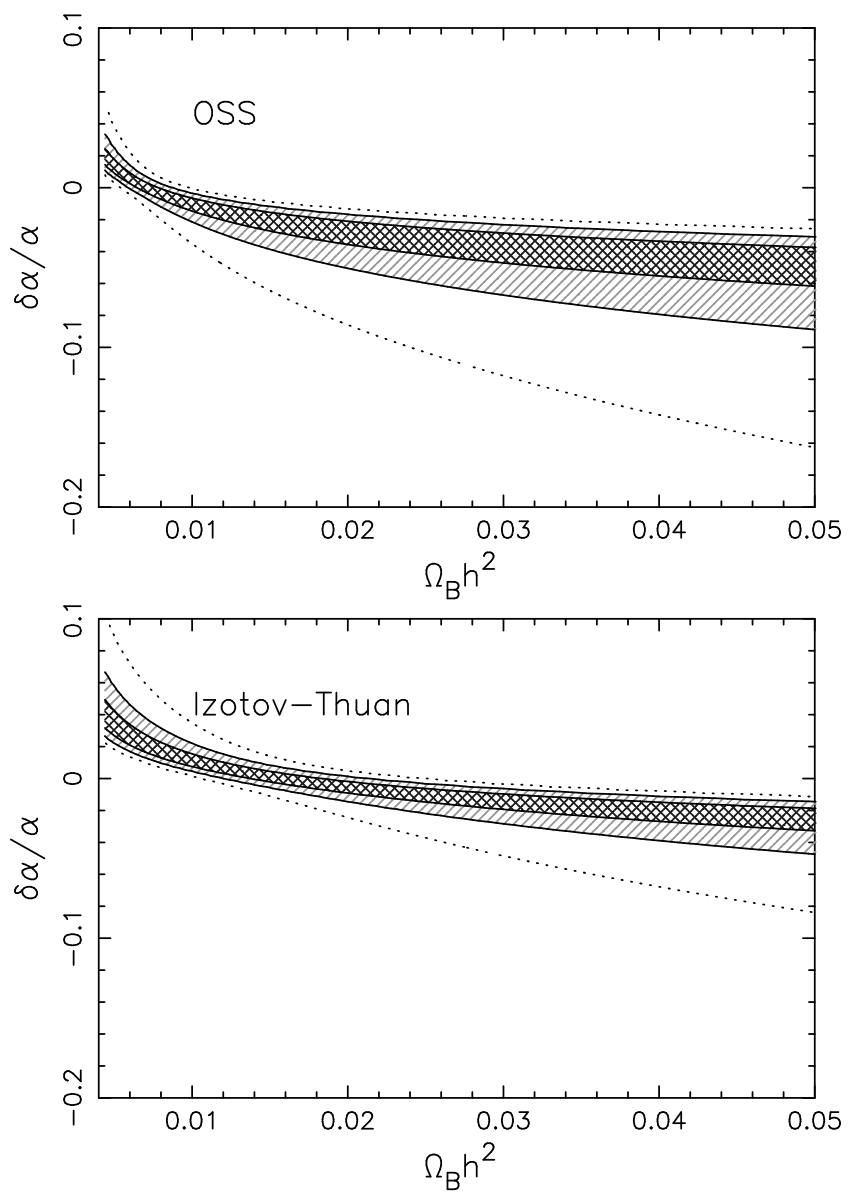

FIG. 6: Regions of $1 \sigma, 2 \sigma$, and $3 \sigma$ confidence, based on the $Y_{P}$ value from OSS 50] (top) and IT [51] (bottom).

the primordial ${ }^{4}$ He mass fraction: Olive, Skillman, and Steigman (OSS) find $Y_{P}=0.234 \pm 0.002$ 50 while Izotov and Thuan (IT) find $Y_{P}=0.244 \pm 0.002$ [51]. The two resulting curves are shown in Fig. 6. It seems clear from these curves that if agreement could be reached concerning the value of $Y_{P}$, quite stringent constraints could be placed on $\delta$ irrespective of $\Omega_{B}$. However, this impression is illusory because such constraints involve the size of the electromagnetic contribution to the nucleon mass splitting. As discussed above, this number is difficult to calculate, and subject to large uncertainty. The confidence intervals shown include allowances for a $30 \%$ Gaussian uncertainty in this parameter (the source of the asymmetric intervals).

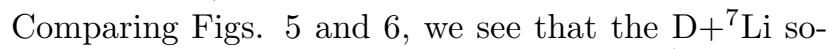
lution above fails the consistency check with ${ }^{4} \mathrm{He}$. Making this solution work would require either $Y_{P}=0.354-$ substantially larger than even the solar value of 0.275 - or else $c=0.045$ (at $Y_{P}=0.244$ ) or $c=0.10$ (at $\left.Y_{P}=0.234\right)$, compared to the current best guess of $c=-0.59$. While $c$ is very uncertain, it cannot be positive if the electromagnetic force contributes to the baryon masses only through self-energies and interactions among constituent quarks [36]. Put another way, a $\chi^{2}$ fit includ- ing all three abundances gives a minimum $\chi^{2}$ far from the $\mathrm{D} / \mathrm{H}+{ }^{7} \mathrm{Li}$ solution (because of the small error bars on $Y_{P}$ ), but the likelihood at this minimum is low: a fit using the IT $Y_{P}$ is rejected at $97.5 \%$ confidence (actually worse than if $\delta=0$ is assumed, since the extra parameter removes a degree of freedom), while a fit using the OSS $Y_{P}$ is rejected at $98.0 \%$ confidence. The exact numerical values of these confidence limits are probably not meaningful, since the $Y_{P}$ error bars in particular are smaller than the systematic uncertainties, but the indication is in any case that varying $\alpha$ does not describe the data significantly better than leaving it fixed.

There is thus no solution favored by all three observed primordial abundances, even at $\delta=0$. Perhaps one of the pairwise combinations of abundances provides an acceptable solution that requires only plausible systematic problems with the third observed abundance. Because the $\mathrm{D}+{ }^{7} \mathrm{Li}$ solution requires such a stretch to match the ${ }^{4} \mathrm{He}$ observations, we can probably rule it out. The $\mathrm{D}+{ }^{4} \mathrm{He}$ case is interesting because it does not require an "extreme" value for $\delta$. This is in the sense that existing claims for time variation in $\alpha$ based on metal lines in quasar absorption systems are on the order of $10^{-5}$. The best fits for BBN with $\mathrm{D}$ and ${ }^{4} \mathrm{He}$ only are at $\Omega_{B} h^{2}=0.020 \pm 0.005, \delta=-0.007_{-0.017}^{+0.010}$ (IT) and $\Omega_{B} h^{2}=0.020_{-0.004}^{+0.005}, \delta=-0.03_{-0.04}^{+0.01}$ (OSS; all errors $2 \sigma$ ). These solutions have the advantage of agreeing with the value of $\Omega_{B} h^{2}$ derived from observations of the cosmic microwave background (CMB). However, ${ }^{7} \mathrm{Li} / \mathrm{H}$ is discrepant by a factor of $2-3$, so this is no better than the standard $\alpha_{\mathrm{BBN}}=\alpha_{0}$ case in its need for a greatly underestimated primordial ${ }^{7} \mathrm{Li} / \mathrm{H}$.

The final pair of abundances available is ${ }^{4} \mathrm{He}+{ }^{7} \mathrm{Li}$. Any ${ }^{4} \mathrm{He}$ constraint crosses both curves defined by the ${ }^{7} \mathrm{Li}$ constraint, and either of the two ${ }^{4} \mathrm{He}$ data sets could be correct. There are thus four different solutions we could consider, but it should suffice to say that they all favor $|\delta|<0.02$ and $\Omega_{B} h^{2}<0.017$. The solution least discrepant from the $\mathrm{D} / \mathrm{H}$ data requires $\mathrm{D} / \mathrm{H}=7.3 \times 10^{-5}$, more than twice the current best observational average and greater than the highest value presently claimed.

Thus, given the present observational data for lightelement abundances, allowing $\alpha$ to vary does not allow $\mathrm{BBN}$ to fit the data in a way that is convincingly better than in the simplest $\alpha_{\mathrm{BBN}}=\alpha_{0}$ case. The existing discrepancies between the light-nuclide abundances and the detailed predictions of BBN are better-explained in terms of systematic effects than in terms of varying $\alpha$. The above analysis produces two plausible constraints on $\alpha_{\mathrm{BBN}}$ : one based on deuterium that derives its strength from the simplicity of the $\mathrm{D} / \mathrm{H}$ measurement (Fig. 3), and one based on ${ }^{4} \mathrm{He}$ that derives its strength from the presumptive strong dependence of $Y_{P}$ on $\alpha_{\mathrm{BBN}}$ (Fig. 6). The constraint based on ${ }^{4} \mathrm{He}$ which was derived in BIR remains the best that one can do to constrain $\alpha_{\mathrm{BBN}}$ independent of any other parameter, with the caveat that the nucleon mass splitting is not well-understood, and so the constraint could be much weaker than it appears. 
The constraint based on $\mathrm{D} / \mathrm{H}$ is perhaps more secure because the varying- $\alpha \mathrm{D} / \mathrm{H}$ yields depend on relatively well-understood physics and because the $\mathrm{D} / \mathrm{H}$ measurement is in principle relatively free of systematic pitfalls: it involves demonstrably pristine material, and it does not depend on radiative transfer or ionization corrections. The deuterium constraint may be expressed as $\log _{10} \Omega_{B} h^{2}-\delta=-1.68 \pm 0.07$ at $2 \sigma$, but it has the disadvantage that one can obtain large variations in $\alpha$ merely by adjusting $\Omega_{B}$. Given the large regions of high confidence level in Fig. 4, ${ }^{7} \mathrm{Li}$ does not provide a useful constraint by itself.

We note that the joint constraint from $\mathrm{D} / \mathrm{H}$ and the IT value of $Y_{P}$ was also examined in Ref. 37. These authors obtained a result of $\delta=-0.007 \pm 0.009$ (compared with our $\delta=-0.007_{-0.017}^{+0.010}$ for this case), and identical limits on $\Omega_{B} h^{2}$ to those found here. One would expect differing values of $\delta$ between their analysis and ours: in our calculation, $\mathrm{D} / \mathrm{H}$ depends more weakly on $\delta$, and the updated laboratory cross section for the crucial $d(p, \gamma)^{3} \mathrm{He}$ cross section is lower than what they used. It appears that these two effects have cancelled by chance at the best-fit solution. $\left({ }^{4} \mathrm{He}\right.$ should behave the same way in both calculations, because we have used the same value of $c$ ). The differing error limits result from a combination of the weaker dependence of $d(p, \gamma)^{3} \mathrm{He}$ on $\delta$ in our treatment, the inclusion of a $9 \%$ cross section uncertainty on our predicted $\mathrm{D} / \mathrm{H}$, and the $30 \%$ uncertainty that we have assigned to $c$.

Finally, an independent constraint on $\Omega_{B} h^{2}$ now exists from measurements of $\mathrm{CMB}$ anisotropies. The current results are $\Omega_{B} h^{2}=0.022 \pm 0.003$ from BOOMERANG [52], $\Omega_{B} h^{2}=0.022_{-0.003}^{+0.004}$ from DASI [53], and $\Omega_{B} h^{2}=$ $0.033 \pm 0.007$ from MAXIMA [54] (all $1 \sigma$ errors). A single direct measurement of $\Omega_{B} h^{2}$ is a vertical line in Figs. 3 6. Values of $\alpha_{\mathrm{BBN}}$ based on these measurements and the individual light-nuclide abundances are shown in Table IV. A firm value of $\delta$ may not be derived from this table, because the relative reliabilities of the inferred primordial abundances are not known. However, a general trend is clear: only the combination ${ }^{7} \mathrm{Li} / \mathrm{H}+$ MAXIMA or the combination of OSS ${ }^{4} \mathrm{He}$ with any of the CMB data provides any strong indication of $\delta \neq 0$. For all other data, the $2 \sigma$ limits either include $\delta=0$ or nearly include it (missing by $10 \%$ or less of the best-fit value).

In principle, the estimation of $\Omega_{B} h^{2}$ from CMB measurements also depends on the value of $\alpha$ at matterradiation decoupling [55, 56, 57], $\alpha_{\mathrm{CMB}}$. This may be included among the parameters to be determined jointly from the CMB, or some value may be assumed as a prior. The results quoted above and used to derive Table IV are based on the assumption that $\alpha_{\mathrm{CMB}}=\alpha_{0}$. The values of $\alpha_{\mathrm{CMB}}$ and $\alpha_{\mathrm{BBN}}$ may have nothing to do with each other: $\alpha$ may evolve between these events. However, barring entropy increases after BBN, the same $\Omega_{B} h^{2}$ today should be inferred from both BBN and the CMB. After marginalizing over $\alpha$, Avelino et al. 37 find from a combination of the BOOMERANG, DASI,
TABLE IV: Constraints obtained by combinining individual light-element abundances with $\Omega_{B} h^{2}$ as inferred from three sets of data on acoustic oscillations in the CMB, and from the analysis of Avelino et al. [37], which marginalized over $\alpha$. Numbers shown are the best-fit $\delta$ and the $\delta$ at the $\pm 2 \sigma$ boundaries.

\begin{tabular}{llllr}
\hline \hline Abundance & CMB experiment & $-2 \sigma$ & $\delta$ & $+2 \sigma$ \\
\hline${ }^{4} \mathrm{He}$ (Izotov) & MAXIMA & -0.053 & -0.017 & -0.0028 \\
& DASI & -0.031 & -0.0083 & 0.0045 \\
& BOOMERANG & -0.030 & -0.0083 & 0.0027 \\
& Marginalized & -0.011 & -0.0052 & 0.0054 \\
${ }^{4} \mathrm{He}$ (OSS) & MAXIMA & -0.12 & -0.039 & -0.020 \\
& DASI & -0.087 & -0.030 & -0.015 \\
& BOOMERANG & -0.086 & -0.030 & -0.016 \\
& Marginalized & -0.078 & -0.027 & -0.014 \\
$\mathrm{D} / \mathrm{H}$ & & & & \\
& MAXIMA & -0.051 & 0.22 & $0.3+{ }^{a}$ \\
& DASI & -0.18 & 0.027 & 0.20 \\
& BOOMERANG & -0.14 & 0.027 & 0.18 \\
& Marginalized & -0.17 & -0.038 & 0.15 \\
${ }^{7} \mathrm{Li} / \mathrm{H}$ & MAXIMA & 0.094 & 0.22 & $0.3+{ }^{a}$ \\
& DASI & -0.003 & 0.18 & $0.3+{ }^{a}$ \\
& BOOMERANG & 0.022 & 0.18 & $0.3+{ }^{a}$ \\
& Marginalized & -0.011 & 0.149 & $0.3+{ }^{a}$ \\
& & & & \\
\hline
\end{tabular}

${ }^{a_{2} \sigma}$ upper boundary exceeds validity of model.

and COBE data that $\Omega_{B} h^{2}=0.019_{-0.002}^{+0.004}$ at $1 \sigma$. For each BBN nuclide, Table IV shows the effects of combining the BBN constraint with this value of $\Omega_{B} h^{2}$. The results are not markedly different than for the DASI and BOOMERANG results independently. This is not surprising, because Avelino et al. 37 find $\alpha_{\mathrm{CMB}}=\alpha_{0}$, within the uncertainties.

\section{CONCLUSIONS}

We have improved the calculation of primordial abundances in varying- $\alpha$ scenarios by including corrections previously neglected: normalization of initial-state Coulomb penetrabilities, final-state Coulomb interactions, photon coupling to nuclear currents, photon energy and barrier penetration in external direct captures, and electromagnetic contributions to the nuclear masses. These affect the interactions between composite nuclei, so they affect all of the BBN abundances except that of ${ }^{4} \mathrm{He}$. In sum, these corrections can amount to half of the effect previously computed for ${ }^{7} \mathrm{Li} / \mathrm{H}$ and about $1 / 3$ of the previous effect on $\mathrm{D} / \mathrm{H}$. The most important corrections are the initial-state penetrability and currentcoupling contributions. The nuclear mass modifications become important only for $\Omega_{B} h^{2}>0.036$. BBN is less sensitive to $\alpha$ than previous calculations indicated, but the sign of the dependence remains the same.

Applying our calculation to the data, the most secure 
constraint we arrive at is $\log _{10} \Omega_{B} h^{2}-\delta=-1.68 \pm 0.07$ $(2 \sigma)$, based on the $\mathrm{D} / \mathrm{H}$ measurements of Tytler and collaborators 40]. Combining this constraint with a constraint $\Omega_{B} h^{2}=0.022 \pm 0.003$ from CMB anisotropies yields $\delta=0.03 \pm 0.07(1 \sigma)$. A separate constraint, subject to both theoretical and astronomical uncertainties which are difficult to assess, is provided by ${ }^{4} \mathrm{He}$ measurements: the BIR result of approximately $|\delta|<0.1$ stands as the best that can be done here, about the same as the $\mathrm{D} / \mathrm{H}+\mathrm{CMB}$ constraint.

Other recent work on time-varying $\alpha$ suggests that between redshifts 1 and $3, \alpha$ is smaller than $\alpha_{0}$ by $\delta=(-0.72 \pm 0.18) \times 10^{-5}$ [2, 3, 国]. Sensitivity to deviations from zero at this level cannot be reached with BBN, for both astronomical and nuclear-physics reasons. Combining this with the large difference in redshifts be- tween $\mathrm{BBN}\left(z \sim 10^{9}\right)$ and the observed absorption systems $(1<z<3)$, it is doubtful that BBN can shed much light on this claim without a specific model for variation of $\alpha$.

\section{Acknowledgments}

We thank Richard Battye and Marc Kamionkowski for helpful conversations, and R. B. Wiringa for providing the EM contributions to the light-nuclide masses. REL was funded by a PPARC Advanced Fellowship. KMN was supported by DoE grant DE-FG03-92-ER40701 and NASA grant NAG5-11725.
[1] J. K. Webb, V. V. Flambaum, C. W. Churchill, M. J. Drinkwater, and J. D. Barrow, Phys. Rev. Lett. 82, 884 (1999).

[2] M. T. Murphy, J. K. Webb, V. V. Flambaum, V. A. Dzuba, C. W. Churchill, J. X. Prochaska, J. D. Barrow, and A. M. Wolfe, Mon. Not. Roy. Astron. Soc. 327, 1208 (2001).

[3] M. T. Murphy, J. K. Webb, V. V. Flambaum, J. X. Prochaska, and A. M. Wolfe, Mon. Not. Roy. Astron. Soc. 327, 1237 (2001).

[4] J. K. Webb, M. T. Murphy, V. V. Flambaum, V. A. Dzuba, J. D. Barrow, C. W. Churchill, J. X. Prochaska, and A. M. Wolfe, Phys. Rev. Lett. 87, 091301 (2001).

[5] J. F. Donoghue, hep-ph/0101130 .

[6] T. Banks, M. Dine, and M. Douglas, Phys. Rev. Lett. 88, 131301 (2002).

[7] J. D. Bekenstein, Phys. Rev. D 25, 1527 (1982).

[8] J. D. Barrow, H. B. Sandvik, and J. Maguijo, Phys. Rev. D 65, 063504 (2002).

[9] E. Kolb, M. Perry, and T. Walker, Phys. Rev. D 33, 869 (1986).

[10] J. D. Barrow, Phys. Rev. D 35, 1805 (1987).

[11] B. A. Campbell and K. A. Olive, Phys. Lett B 345, 429 (1995).

[12] L. Bergström, S. Iguri, and H. Rubinstein, Phys. Rev. D 60, 045005 (1999), abbreviated BIR.

[13] P. Langacker, G. Segrè, and M. J. Strassler, Phys. Lett. B 528, 121 (2002).

[14] T. Dent and M. Fairbairn, astro-ph/0112279 .

[15] S. R. Beane and M. J. Savage, hep-ph/0206113 .

[16] X. C. Calmet and H. Fritzsch, hep-ph/0112110 .

[17] M. Abramowitz and I. A. Stegun, eds., Handbook of Mathematical Functions (Dover, New York, 1965).

[18] J. M. Blatt and V. F. Weisskopf, Theoretical Nuclear Physics (Springer, New York, 1979).

[19] R. F. Christy and I. Duck, Nucl. Phys. 24, 89 (1961).

[20] K. M. Nollett, Phys. Rev. C 63, 054002 (2001).

[21] B. Buck, R. A. Baldock, and J. A. Rubio, J. Phys. G 11, L11 (1985).

[22] B. S. Pudliner, V. R. Pandharipande, J. Carlson, S. C. Pieper, and R. B. Wiringa, Phys. Rev. C 56, 1720 (1997).

[23] R. B. Wiringa, private communication.
[24] M. S. Smith, L. H. Kawano, and R. A. Malaney, Astrophys. J. Suppl. 85, 219 (1993), abbreviated SKM.

[25] G. R. Caughlan and W. A. Fowler, At. Data Nucl. Data Tables 40, 283 (1988).

[26] G. J. Schmid, B. J. Rice, R. M. Chasteler, M. A. Godwin, G. C. Kiang, L. L. Kiang, C. M. Laymon, R. M. Prior, D. R. Tilley, and H. R. Weller, Phys. Rev. C 56, 2565 (1997).

[27] L. Ma, H. J. Karwowski, C. R. Brune, Z. Ayer, T. C. Black, J. C. Blackmon, E. J. Ludwig, M. Viviani, A. Kievsky, and R. Schiavilla, Phys. Rev. C 55, 588 (1997).

[28] C. R. Brune, R. W. Kavanagh, and C. Rolfs, Phys. Rev. C 50, 2205 (1994).

[29] C. Brune, K. I. Hahn, R. W. Kavanagh, and P. R. Wrean, Phys. Rev. C 60, 015801 (1999).

[30] C. Angulo et al., Nucl. Phys. A656, 3 (1999).

[31] K. M. Nollett and S. Burles, Phys. Rev. D 61, 123505 (2000).

[32] R. Lopez and M. S. Turner, Phys. Rev. D 59, 103502 (1999).

[33] T. M. Bania, R. T. Rood, and D. S. Balser, Nature 415, 54 (2002).

[34] L. H. Kawano, Fermilab-pub-88/34-a (1988), (unpublished).

[35] L. H. Kawano, Fermilab-pub-92/04-a (1992), (unpublished).

[36] J. Gasser and H. Leutwyler, Phys. Rep. 87, 77 (1982).

[37] P. P. Avelino, S. Esposito, G. Mangano, C. J. A. P. Martins, A. Melchiorri, G. Miele, O. Pisanti, G. Rocha, and P. T. P. Viana, Phys. Rev. D 64, 102505 (2001).

[38] K. A. Olive, G. Steigman, and T. P. Walker, Phys. Rept. 389, 333 (2000).

[39] D. Tytler, J. M. O'Meara, N. Suzuki, and D. Lubin, Physica Scripta 85, 12 (2000).

[40] J. M. O'Meara, D. Tytler, D. Kirkman, N. Suzuki, J. X. Prochaska, D. Lubin, and A. M. Wolfe, Astrophys. J. 552, 718 (2001).

[41] M. Pettini and D. V. Bowen, Astrophys. J. 560, 41 (2001).

[42] S. D'Odorico, M. Dessauges-Zavadsky, and P. Molaro, Astron. Astrophys. 368, L21 (2001). 
[43] S. A. Levshakov, M. Dessauges-Zavadsky, and S. D. D'Odorico, Astrophys. J. 565, 696 (2002).

[44] S. Ryan, Proc. IAU Symposium 198, "The Light Elements and Their Evolution" (Astronomical Society of the Pacific, 2000), p. 249.

[45] S. G. Ryan, T. C. Beers, K. A. Olive, B. D. Fields, and J. E. Norris, Astrophys. J. 530, L57 (2000).

[46] S. Vauclair and C. Charbonnel, Astron. Astrophys. 295, 715 (1995).

[47] M. H. Pinsonneault, T. P. Walker, G. Steigman, and V. K. Narayanan, Astrophys. J. 527, 180 (1999).

[48] M. Salaris and A. Weiss, Astron. Astrophys. 376, 955 (2001).

[49] M. Asplund, in L. da Silva, M. Spite, and J. R. de Medeiros, eds., The Light Elements and Their Evolution (Astronomical Society of the Pacific, Provo, 2000),
IAU Symp. 198.

[50] K. Olive, G. Steigman, and E. Skillman, Astrophys. J. 483, 788 (1998).

[51] Y. Izotov and T. Thuan, Astrophys. J. 500, 188 (1998).

[52] C. B. Netterfield et al., Astrophys. J. (2002), in press; astro-ph/0104460.

[53] C. Pryke, N. W. Halverson, E. M. Leitch, J. Kovac, J. E. Carlstrom, W. L. Holzapfel, and M. Dragovan, Astrophys. J. 568, 46 (2002).

[54] R. Stompor et al., Astrophys. J. 561, L7 (2001).

[55] M. Kaplinghat, R. Scherrer, and M. Turner, Phys. Rev. D 60, 023516 (1999).

[56] S. Hannestad, Phys. Rev. D 60, 023515 (1999).

[57] R. A. Battye, R. Crittenden, and J. Weller, Phys. Rev. D 63, 043505 (2001). 